PHYSICAL REVIEW B 92, 104203 (2015)

\title{
Collective excitations in liquid and glassy 3-methylpentane
}

\author{
Paola Benassi and Michele Nardone \\ Dipartimento di Scienze Fisiche e Chimiche, Università degli Studi dell'Aquila, 67100 L'Aquila, Italy
}

Andrea Giugni

King Abdullah University of Science and Technology, PSE and BESE Divisions, Thuwal, 23955-6900, Saudi Arabia

\author{
Giacomo Baldi* \\ IMEM-CNR, Parco Area delle Scienze 37/A, 43124 Parma, Italy
}

Aldo Fontana

Dipartimento di Fisica, Università di Trento, 38050 Povo (Trento), Italy

(Received 13 April 2015; revised manuscript received 4 August 2015; published 28 September 2015)

\begin{abstract}
We present a detailed investigation of the terahertz vibrational dynamics of 3-methylpentane performed by means of high-resolution inelastic x-ray scattering (IXS). We probe the dynamics in a large temperature range, which includes the glass, the supercooled liquid, and the liquid phases. The characteristic frequency of the excitations follows a well-defined dispersion curve extending beyond $8 \mathrm{~nm}^{-1}$ at all the investigated temperatures, indicating the persistence of a solidlike behavior also in the liquid phase. This implies the existence of a pseudo-Brillouin zone whose size compares surprisingly well with the periodicity inferred from the first sharp diffraction peak in the static structure factor. We show that, in the investigated temperature range, both sizes undergo a variation of about $15 \%-20 \%$, comparable to that of the average intermolecular distance. We finally show that the IXS sound velocity coincides with the infinite frequency sound velocity previously inferred from visible and ultraviolet Brillouin spectroscopy data. This analysis confirms the role of the shear relaxation processes in determining the variation with frequency of the apparent sound velocity.
\end{abstract}

DOI: 10.1103/PhysRevB.92.104203

PACS number(s): 63.50.Lm, 64.70.kj, 61.05.C-, 61.43.Fs

\section{INTRODUCTION}

The existence of propagating collective excitations at large exchanged wave-vector values in systems which do not possess translational order, such as amorphous solids and liquids, has been widely demonstrated by inelastic x-ray scattering [1-5] and by neutron scattering experiments [6-9]. These excitations exhibit a dispersion relation which closely resembles that of longitudinal modes of a crystalline solid, extending in some cases even beyond what we can refer to as a pseudo-Brillouin zone. The boundary of this pseudo-Brillouin zone generally occurs at wave vectors roughly one half of the first maximum $q_{m}$ of the static structure factor $S(q)$ [10].

In this paper, we report measurements of inelastic $\mathrm{x}$-ray scattering (IXS) spectra of 3-methylpentane (3MP) at different exchanged wave vectors $q$ extending up to $q_{m}$ and covering the temperature range from room temperature down to the glass transition. Such measurements will be analyzed in order to characterize the behavior of the collective excitations and their relationship with the propagation of sound in this glassforming liquid.

$3 \mathrm{MP}$ is a structural isomer of hexane, molecular formula $\mathrm{C}_{6} \mathrm{H}_{14}$, formed by a pentane chain with a methyl group bonded to the third carbon atom. It belongs to a group of molecules referred to as branched-chain alkanes [11] or as methylated alkanes [12]. It is a nonpolymeric glass-forming liquid with a calorimetric glass transition temperature at $T_{G} \approx 77 \mathrm{~K}$ [13-15]. The glassy phase is readily formed by cooling the

\footnotetext{
*giacomo.baldi@cnr.it
}

liquid below the glass transition temperature, thus allowing us to study the behavior of both liquid and disordered solid phases. Because of the relatively small atomic number $Z$, it can be successfully measured in an IXS experiment.

It will be shown that the analysis of the spectra yields dispersion curves which are identifiable also above the pseudoBrillouin zone boundary, not only in the glassy phase but also in the liquid, indicating the persistence of a solidlike behavior. These dispersion curves exhibit maxima which are found to fall close to $q_{m} / 2$ [as obtained from measurements of the structure factor $S(q)$ ] at all temperatures, yielding a pseudo-lattice parameter $a_{0}$ which increases monotonically with temperature.

The apparent sound velocity, as obtained from the initial slope of these curves, favorably compares with the highfrequency sound velocities inferred from visible and ultraviolet Brillouin spectroscopy [16]. This comparison confirms the role of shear relaxation processes in determining the difference between zero frequency and infinite frequency sound velocities in this system.

\section{EXPERIMENTAL DETAILS AND DATA ANALYSIS}

The sample (3MP $>99 \%$ purity) supplied by Sigma Aldrich, was contained in an aluminium vessel equipped with high-transmittance Kapton windows which contribute negligibly to the collected scattered intensity.

The spectral measurements were performed at the high-energy resolution beam line ID16 of the European Synchrotron Radiation Facility in Grenoble, France. The X-ray beam (incident energy $E_{0}=21747 \mathrm{eV}$ ) impinges on the sample and the scattered radiation is analyzed by means of a 


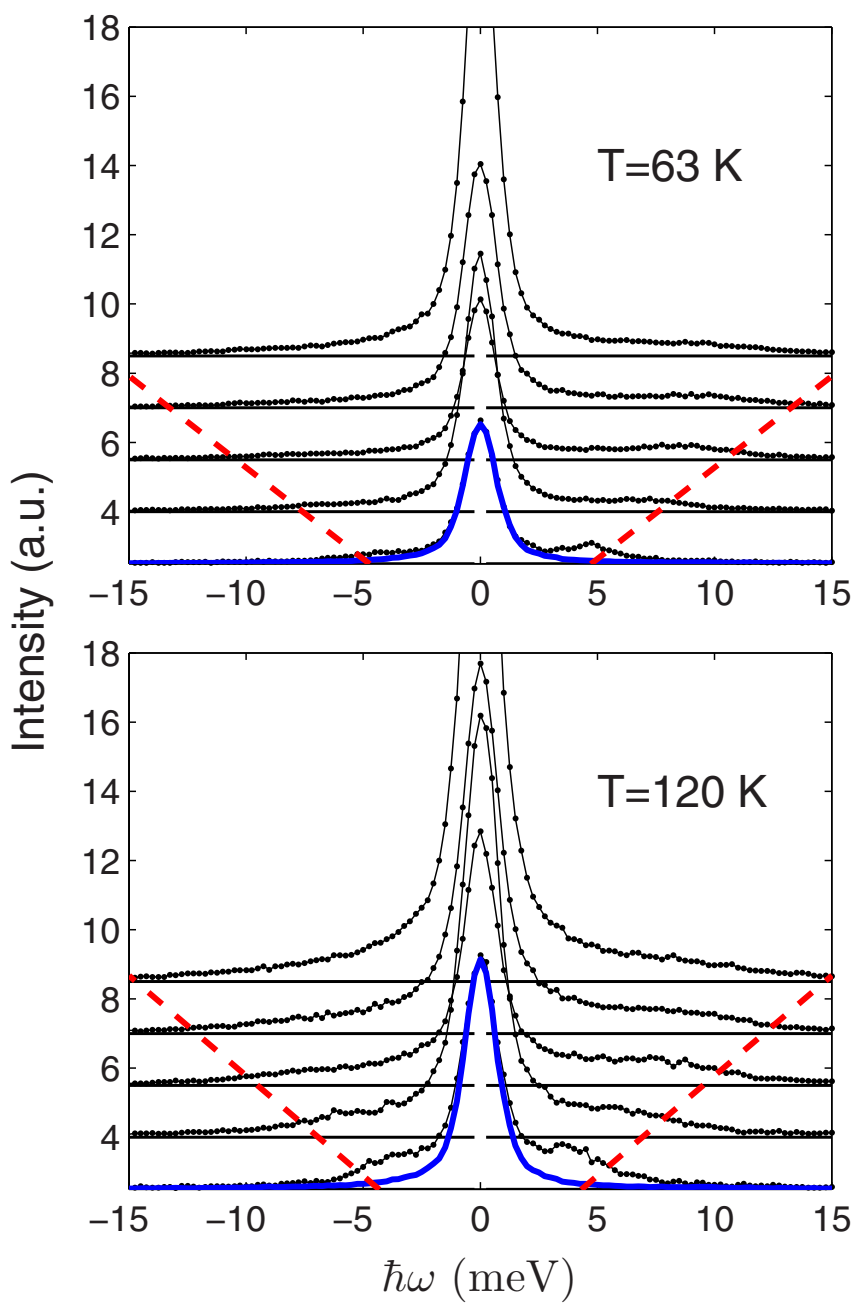

FIG. 1. (Color online) Selection of IXS spectra (black dots) as measured at $T=120 \mathrm{~K}$ in the liquid phase (bottom panel) and at $T=63 \mathrm{~K}$ in the glassy phase (top panel). The spectra are vertically shifted by an amount proportional to the exchanged wave vector (from bottom to top, $q=2.5,4.0,5.5,7.0$, and $8.5 \mathrm{~nm}^{-1}$ ). The full line (blue) is the measured instrumental transfer function while the dashed line (red) is the linear dispersion obtained using the apparent sound velocity (see discussion).

6.5-m-long arm hosting five crystal analyzers. The exchanged wave vectors are selected by rotating the analyzers arm. Energy scans are performed at constant momentum transfer (between 1 and $14.5 \mathrm{~nm}^{-1}$ in our case) by varying the relative temperatures of the monochromator crystal with respect to the analyzer crystals. Further details on the beam line are reported elsewhere [17]. We measured the overall spectrometer transfer function at each analyzer by measuring the elastic scattering from a poly(methylmethacrylate) (PMMA) sample. The PMMA was cooled at $T=10 \mathrm{~K}$ and measured at the exchanged wave vector $q$ corresponding to the maximum of its structure factor where the inelastic scattering contribution to the spectrum is negligible. The resolution profile $R(\omega)$ of all the analyzers turns out to be essentially Lorentzian with a FWHM of about $1.5 \mathrm{meV}$.

Figure 1 shows, as an example, the IXS experimental data for $T=120 \mathrm{~K}$ in the liquid phase and for $T=63 \mathrm{~K}$ in the

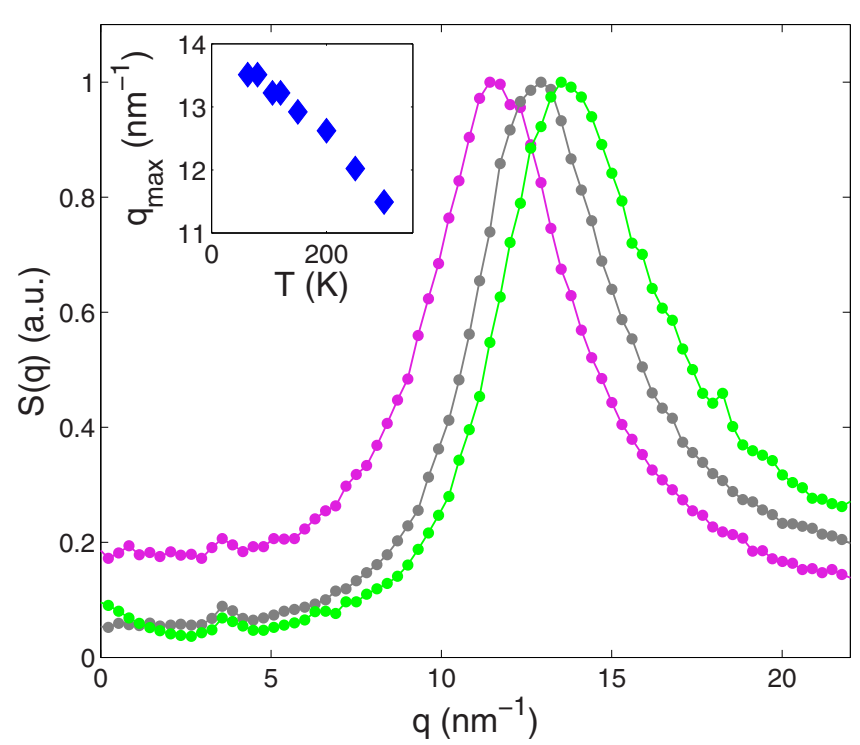

FIG. 2. (Color online) Measured static structure factor $S(q)$ at selected temperatures (from left to right): $T=300 \mathrm{~K}$ (magenta), $T=150 \mathrm{~K}$ (gray), and $T=63 \mathrm{~K}$ (green). The intensities of the data are arbitrarily normalized to their maxima. The inset shows the temperature behavior of the position $q_{\max }$ of the first sharp diffraction peak. These values have been obtained fitting $S(q)$ with a parabola around its maximum.

glassy phase at different $q$ s together with a typical instrumental resolution profile. Every spectrum is obtained by averaging four energy scans, each one lasting typically 45 minutes, after normalization to the intensity of the incident beam.

The total scattered intensity $S(q)$ has also been measured at all temperatures as a function of $q$. Selected $S(q)$ are reported in Fig. 2 with intensities normalized to their maxima. The shift in the position $q_{\text {max }}$ of the first sharp diffraction peak is evident at increasing temperature.

At small wave vectors the spectra show, at all temperatures, evidence of well-defined side peaks which exhibit detailed balance between Stokes and anti-Stokes sides. These side peaks become broader and less prominent as $q$ is increased and are hardly detectable above $10 \mathrm{~nm}^{-1}$, particularly in the liquid phase. All the spectra exhibit a relatively intense central line with a linewidth comparable with that of the resolution profile, even though some broadening can be detected at the highest- $q$ values and high temperature.

On the basis of these evidences, we have analyzed these spectra using a normalized simple damped harmonic oscillator line shape (DHO), assuming

$$
I(\omega) \propto R(\omega) \otimes\left[A \delta(\omega)+S_{\mathrm{DHO}}(q, \omega)\right]+B,
$$

where

$$
S_{\mathrm{DHO}}(q, \omega)=f(\omega, T) \frac{1}{\pi} \frac{\omega_{0}^{2}(q) \Gamma(q)}{\left[\omega^{2}-\omega_{0}^{2}(q)\right]^{2}+\omega^{2} \Gamma^{2}(q)}
$$

and

$$
f(\omega, T)=\frac{\hbar \omega}{K T}\left[\frac{1}{1-\exp \left(-\frac{\hbar \omega}{K T}\right)}\right]
$$



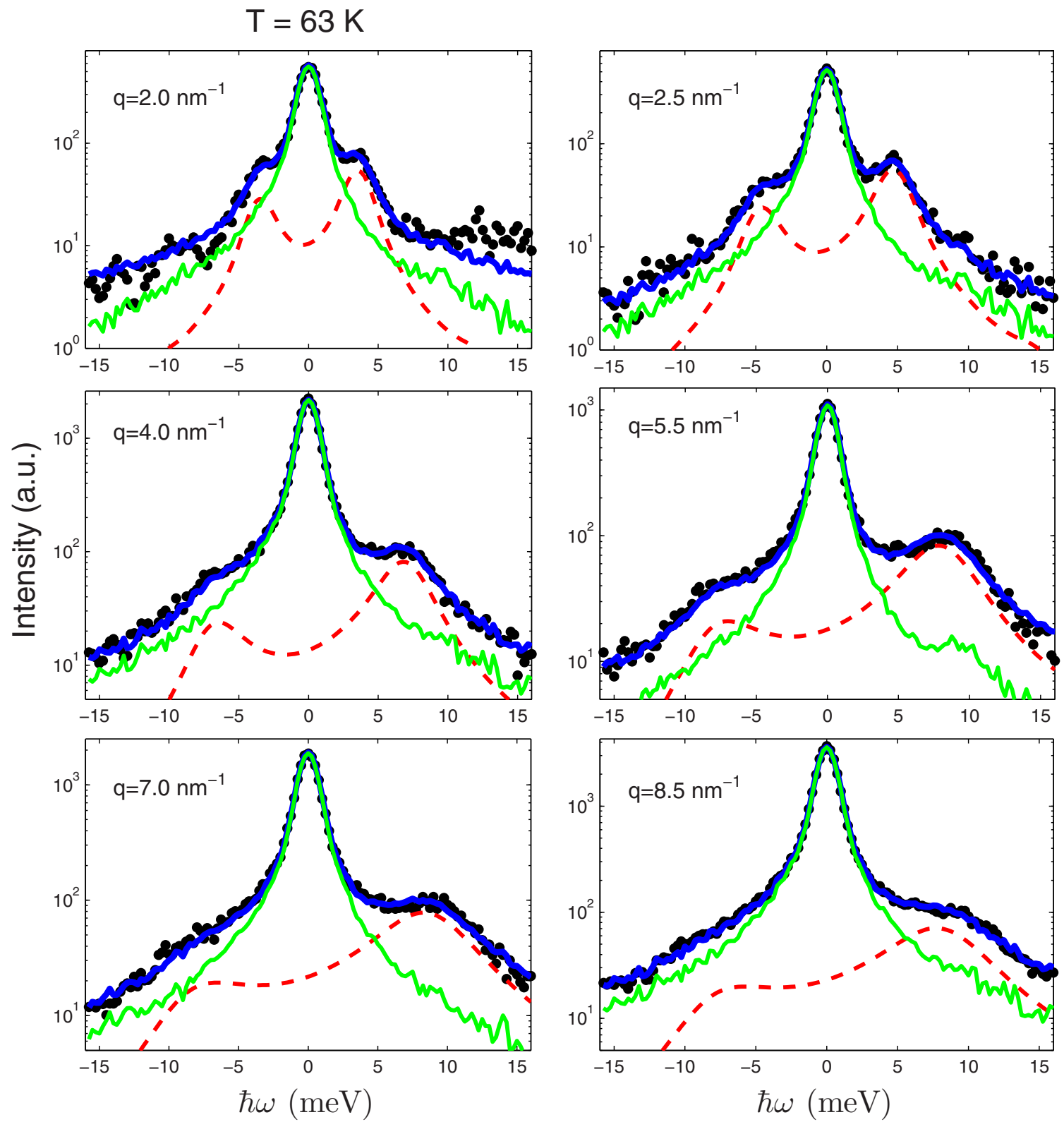

FIG. 3. (Color online) IXS spectra (black dots) measured in the glassy phase ( $T=63 \mathrm{~K}$ ) for selected values of $q$. The logarithmic intensity scale allows one to appreciate the details of the instrumental transfer function (thin green solid line) and of the inelastic contribution of the fitting function (red dashed line). The reconstructed profile $I(\omega)$ is shown as a thick solid line (blue).

accounts for detailed balance. The physically relevant free fit parameters are the frequency $\omega_{0}$, the damping $\Gamma$, and the amplitude $A$ of the elastic contribution relative to the inelastic one. $B$ accounts for a small background of the order of a few counts for each spectrum.

Some typical fits are shown in Fig. 3 for $T=63 \mathrm{~K}$ and in Fig. 4 for $T=120 \mathrm{~K}$ in the $q$ range: $2 \leqslant q \leqslant 8.5 \mathrm{~nm}^{-1}$. The quality of the fits is comparably good at all other temperatures, the $\chi^{2}$ values being always of the order of unity. At high- $q$ values, however, the simple line shape adopted appears to become progressively less adequate in describing the spectra, particularly at high temperatures. For the highest- $q$ values and at high temperatures, the side peaks intensity decreases considerably and the central line slightly broadens. As a consequence, the parameter $A$, the relative intensity of the two spectral contributions, becomes unreliable.

The spectra in Fig. 3 are measured below the glass transition temperature, while those in Fig. 4 are in the liquid, above the melting temperature. The effect of increasing the temperature, already apparent from a visual inspection of the spectra, is to broaden the inelastic features, making the peaks more defined at low temperatures.

\section{RESULTS AND DISCUSSION}

In this paper, we focus our attention mainly on the temperature and wave-vector behavior of the parameter $\omega_{0}$. The values of $\omega_{0}$, as obtained from the fitting of the spectral 

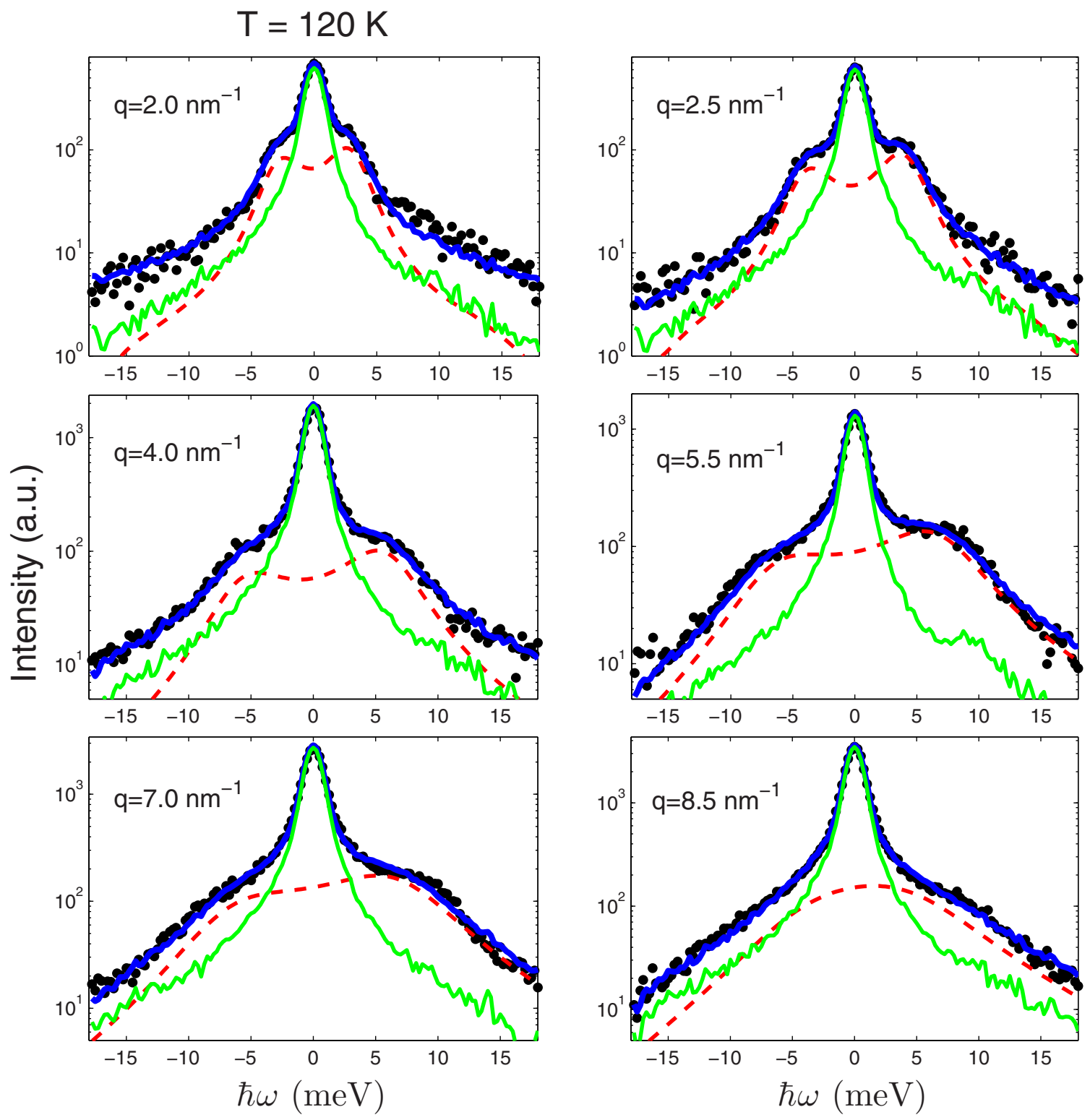

FIG. 4. (Color online) IXS spectra (black dots) measured in the liquid phase $(T=120 \mathrm{~K})$ for selected values of $q$. Symbols as in Fig. 3.

line shapes, are reported in Fig. 5 as a function of $q$ for different temperatures. The values of $\omega_{0}$ exhibit a well-defined linear dispersion at low $q$ s typical of the hydrodynamic behavior. More interestingly, at larger $q \mathrm{~s}$ the dispersion relation presents a maximum, located in the range $6-8 \mathrm{~nm}^{-1}$, which is well defined at all temperatures in both liquid and solid phases.

We have parametrized this behavior using a simple sinusoidal dispersion of the form [18]

$$
E=\hbar \omega=\hbar v \frac{2 q_{0}}{\pi}\left|\sin \left(\frac{\pi q}{2 q_{0}}\right)\right|
$$

which is characterized by an initial slope, determining the apparent sound velocity $v$, being $E \simeq \hbar v q$, and by the occurrence of a maximum for $q=q_{0}$. The $q_{0}$ values can be related to the pseudo-Brillouin zone boundary wave vector characterized by a "lattice parameter" $a_{0}=\pi / q_{0}$ describing the local range order. The parameters $v$ and $q_{0}$ have been determined fitting the data excluding those at the higher wave vector and temperatures, where the spectral fitting procedure appeared, as already mentioned, to be less reliable. Looking at Fig. 5, it should be noted that this very simple model describes surprisingly well the experimental results.

The values of the apparent sound velocity $v$ are shown, as a function of temperature, in Fig. 6, together with those obtained from $90^{\circ}$ Brillouin scattering spectra measured with 266-nm excitation [16], corresponding to exchanged wave vectors of about $0.05 \mathrm{~nm}^{-1}$.

In Fig. 7, we report the values of the pseudo-lattice parameter $a_{0}$ as a function of temperature together with the corresponding values obtained from the first diffraction maximum $q_{\max }$, i.e.,

$$
a_{m}=\frac{2 \pi}{q_{\max }} .
$$

Both $a_{m}$ and $a_{0}$, which are numerically quite similar, exhibit a weak temperature dependence (see Fig. 7) which well 


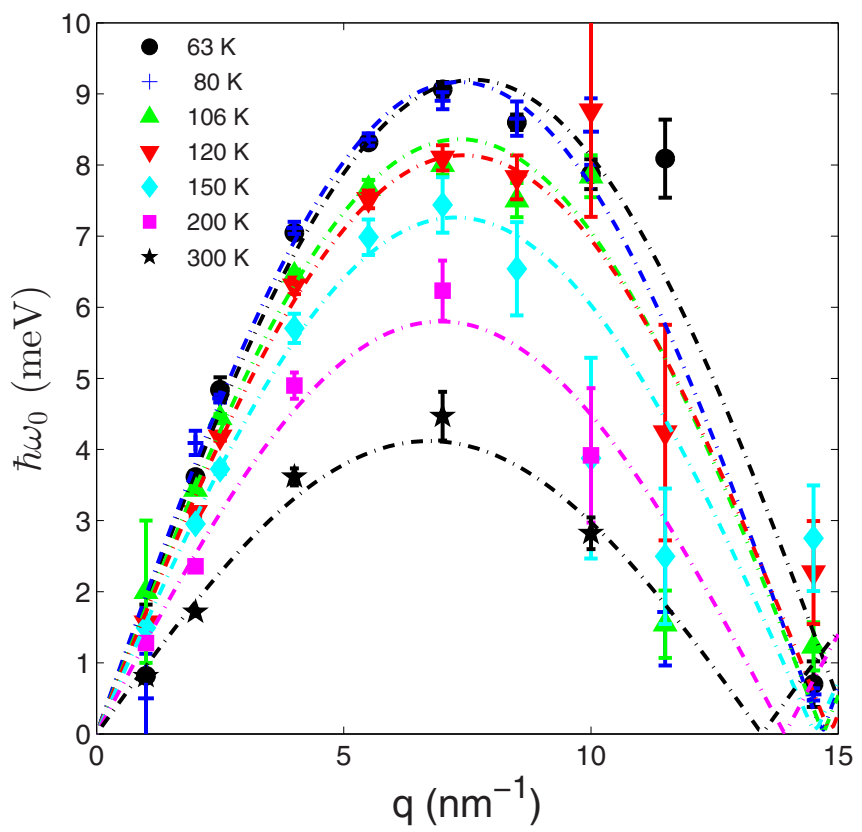

FIG. 5. (Color online) The fitting parameter $\omega_{0}$ versus $q$ (see text) at different temperatures as indicated in the legend. For each temperature, the line is the best fit of Eq. (1) to the data. The high- $q$ data, typically above $8.5 \mathrm{~nm}^{-1}$, have not been included in the fitting procedure.

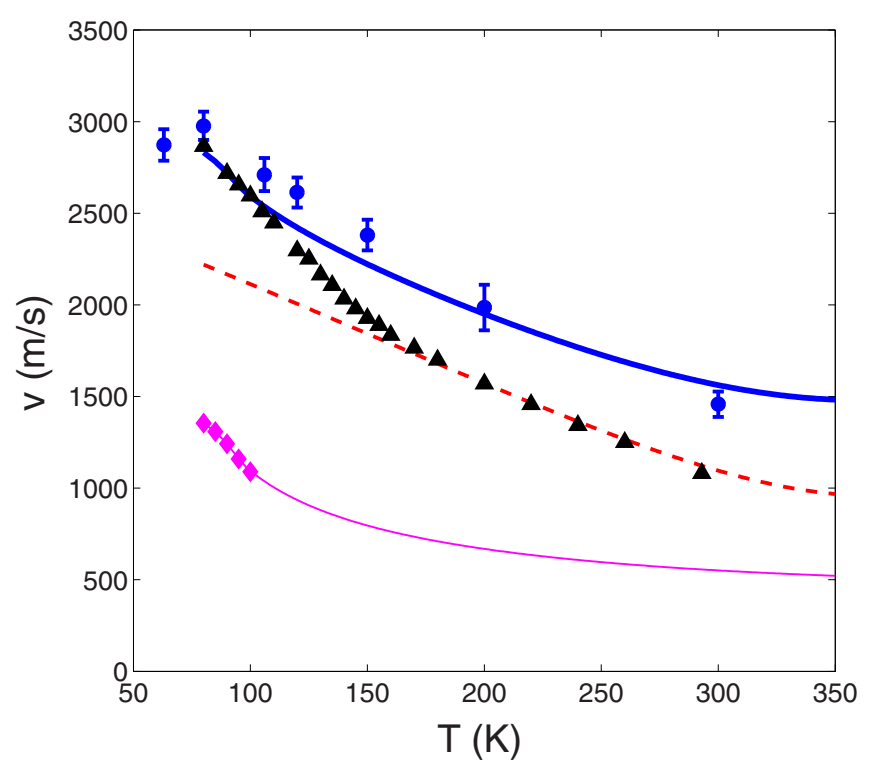

FIG. 6. (Color online) Apparent sound velocity $v$ versus temperature as obtained from IXS measurements (blue dots) and from Brillouin scattering measurements at $90^{\circ}$ with $266-\mathrm{nm}$ excitation (black triangles). The full thick line (blue) and the dashed line (red) represent, respectively, the infinite- and zero-frequency limits, as argued from [16]. Diamonds are the transverse velocities measured by Brillouin scattering at $90^{\circ}$ with 532-nm excitation together with the extrapolation of their high-temperature behavior (thin solid line, magenta) following Barlow [20].

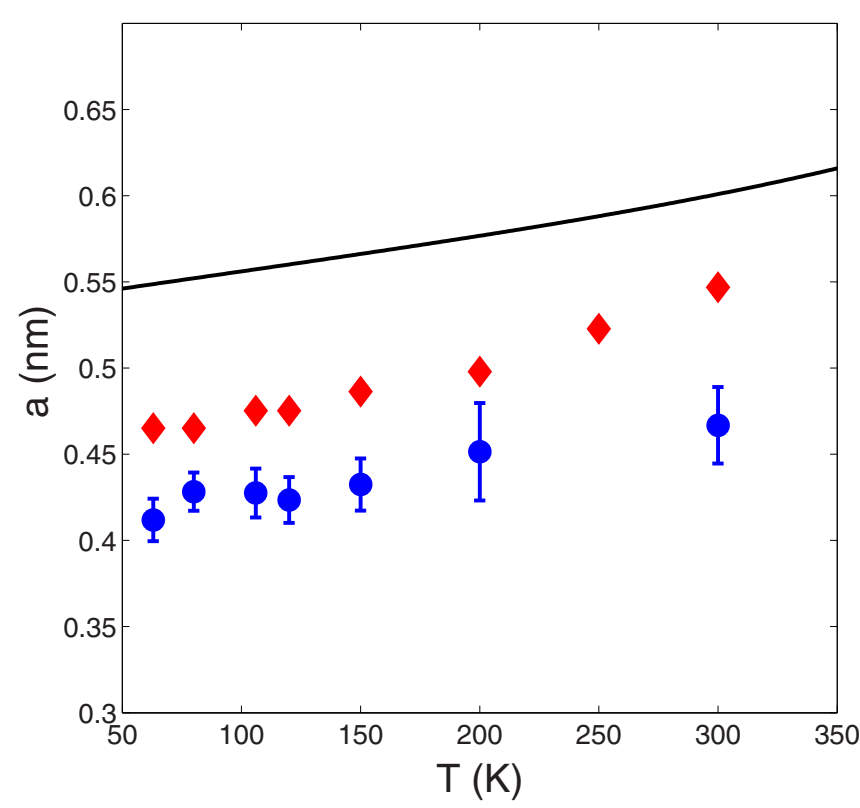

FIG. 7. (Color online) Characteristic length $a$ versus temperature. The $a_{0}$ values (blue full dots) are obtained fitting the dispersion curves (see text) while $a_{m}$ values (red diamonds) are obtained from the maxima of $S(q)$. The full (black) line is obtained from density data (see text).

compares with that of the average intermolecular distance given by

$$
a=\left(\frac{1}{\rho}\right)^{\frac{1}{3}},
$$

$\rho$ being the molecular number density obtained from Ref. [19]. The values of $a_{m}(T)$ appear to be systematically larger than those of $a_{0}(T)$. This could be due to the presence of the intramolecular interference term which has not been taken into account in analyzing diffraction data.

\section{CONCLUSIONS}

The inelastic $\mathrm{x}$-ray scattering spectra from liquid and amorphous solid 3MP reported in this work show propagating collective excitations that are well defined in a rather wide temperature range, extending from room temperature down to the glass transition temperature, and for exchanged wavevector values as large as those of the first sharp diffraction peak. Analyzing the spectra in terms of a simple DHO line shape we have derived dispersion curves from which we have obtained the temperature dependence of the apparent sound velocity $v(T)$ and of the pseudo-lattice parameter $a_{0}(T)$.

The values of $a_{0}(T)$, which have been obtained analyzing dynamic properties of the system, are compared with those of $a_{m}(T)$ derived from purely structural properties. The comparison shows that both quantities increase almost linearly with temperature, following essentially the behavior of the average intermolecular distance $a$, and yielding a variation of about $15 \%-20 \%$, in the entire investigated range. The smooth behavior of both quantities across the glass transition confirms that no severe structural changes occur in this molecular liquid upon vitrification. 
The apparent sound velocity is compared in Fig. 6 with sound velocity values reported in a recent paper [16]. In that paper, some of us performed a thorough analysis of both longitudinal and transverse relaxation processes in fluid 3MP analyzing Brillouin spectra at different scattering angles and different excitation wavelengths (532 and $266 \mathrm{~nm}$ ). It was found that, on the basis of the observed transverse modes velocity (diamonds in Fig. 6), both longitudinal and shear viscosity relaxation processes are required in order to justify the observed linewidths of the longitudinal Brillouin peaks. The identification and characterization of both these processes allowed the authors to obtain the "infinite frequency" sound velocity (full line in the same figure). The apparent sound velocity determined by the longitudinal Brillouin peak position (up triangles in the figure) is seen to coincide with the "infinite frequency" values at temperatures close to $T_{G}$, while, as the temperature is increased, it tends to the "zero frequency" limit (dashed-dotted line) given by ultrasonic measurements. The IXS data of the present work are indeed in very good agreement with these "infinite frequency" values, confirming the validity of the previous analysis.

\section{ACKNOWLEDGMENTS}

We gratefully acknowledge the prolific debates and stimulating discussions with the late Professor M. Sampoli who has brought to our attention the interesting phenomenology of this glass-forming liquid. We acknowledge the European Synchrotron Radiation Facility for provision of synchrotron radiation facilities and we would like to thank F. Albergamo for assistance in using beamline ID16. We acknowledge S. Caponi for the useful discussions.
[1] T. Scopigno, G. Ruocco, and F. Sette, Rev. Mod. Phys. 77, 881 (2005).

[2] A. Matic, C. Masciovecchio, D. Engberg, G. Monaco, L. Börjesson, S. C. Santucci, and R. Verbeni, Phys. Rev. Lett. 93, 145502 (2004).

[3] V. V. Brazkin and K. Trachenko, J. Phys. Chem. B 118, 11417 (2014).

[4] B. Ruzicka, T. Scopigno, S. Caponi, A. Fontana, O. Pilla, P. Giura, G. Monaco, E. Pontecorvo, G. Ruocco, and F. Sette, Phys. Rev. B 69, 100201 (2004).

[5] G. Baldi, M. Zanatta, E. Gilioli, V. Milman, K. Refson, B. Wehinger, B. Winkler, A. Fontana, and G. Monaco, Phys. Rev. Lett. 110, 185503 (2013).

[6] L. E. Bove, E. Fabiani, A. Fontana, F. Paoletti, C. Petrillo, O. Pilla, and I. C. V. Bento, Europhys. Lett. 71, 563 (2005).

[7] E. Fabiani, A. Fontana, and U. Buchenau, J. Chem. Phys. 128, 244507 (2008).

[8] L. Orsingher, G. Baldi, A. Fontana, L. E. Bove, T. Unruh, A. Orecchini, C. Petrillo, N. Violini, and F. Sacchetti, Phys. Rev. B 82, 115201 (2010).

[9] M. Zanatta, A. Fontana, A. Orecchini, C. Petrillo, and F. Sacchetti, J. Phys. Chem. Lett. 4, 1143 (2013).
[10] U. Balucani and M. Zoppi, Dynamics of the Liquid State (Clarendon, Oxford, 1994).

[11] S. Shahriari, A. Mandanici, L.-M. Wang, and R. Richter, J. Chem. Phys. 121, 8960 (2004).

[12] K. L. Ngai, Phys. Rev. B 71, 214201 (2005).

[13] A. C. Ling and J. E. Willard, J. Phys. Chem. 72, 1918 (1968).

[14] H. L. Finke and J. F. Messerly, J. Chem. Thermodyn. 5, 247 (1973).

[15] L. M. Wang, C. A. Angel, and R. Richert, J. Chem. Phys. 125, 074505 (2006).

[16] P. Benassi, M. Nardone, and A. Giugni, J. Chem. Phys. 137, 094504 (2012).

[17] C. Masciovecchio, U. Bergmann, M. H. Krisch, G. Ruocco, F. Sette, and R. Verbeni, Nucl. Instrum. Methods Phys. Res. Sect. B 111, 181 (1996).

[18] C. Kittel, Introduction to Solid State Physics (Wiley, New York, 1966).

[19] A. A. Ruth, B. Nickel, and H. Lesche, Z. Phys. Chem. 175, 91 (1992).

[20] J. Barlow and J. Lamb, Discuss. Faraday Soc. 43, 223 (1967). 